\title{
A STUDY OF \\ MULTISTAGE/MULTIFUNCTION COLUMN FOR FINE PARTICLE SEPARATION
}

\author{
QUARTERLY TECHNICAL PROGRESS REPORT \\ (January 1, 1995 - March 31, 1995)
}

$\begin{array}{ll}\text { Principal Investigator: } & \text { Dr. Shiao-Hung Chiang } \\ \text { Project Officer: } & \text { Dr. Ralph W. Lai }\end{array}$

Dr. Ralph W. Lai

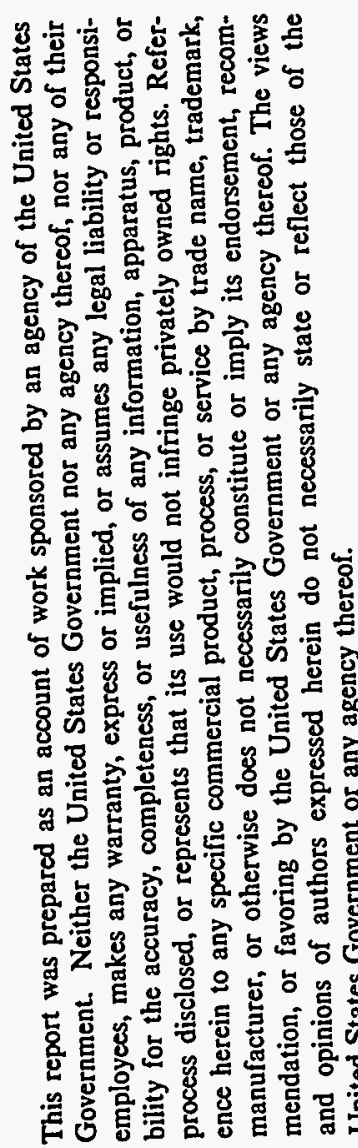

Work Performed under DOE Contract No. DE-PG22-94PC94217

(Award: \$200,000; Duration: July 8, 1994 - July 7, 1997)

Submitted to

U.S. Department of Energy

Pittsburgh Energy Technology Center

Pittsburgh, PA 15236-0940

by

Chemical and Petroleum Engineering Department

University of Pittsburgh

Pittsburgh, PA 15261

April 20, 1995 


\section{DISCLAIMER}

Portions of this document may be illegible in electronic image products. Images are produced from the best available original document. 


\subsection{INTRODUCTION}

The overall objective of the proposed research program is to explore the potential application of a new invention involving a multistage column equipped with vortex-inducing loopflow contactors (hereafter referred to as the multistage flotation column) for flotation process. The research work will identify the design parameters and their effects on the performance of the separation process. The results of this study will provide a basis for further development of this technology.

In the last quarter, we completed equipment design and started to construct the new column for hydrodynamic tests. In this quarter, Task 2 (Equipment Design and Construction) was completed, and the experimental work mainly focused on gas holdup measurement. The program schedule is shown in Table 1.1. A detailed description of technical progress is presented below.

Table 1.1 Project Schedule

\begin{tabular}{|c|c|c|c|c|c|c|c|c|c|c|c|c|c|}
\hline \multirow{2}{*}{ Tasks } & \multicolumn{3}{|c|}{1994} & \multicolumn{3}{|c|}{1995} & \multicolumn{3}{|c|}{1996} & \multicolumn{3}{|c|}{1997} & \multirow[b]{2}{*}{6} \\
\hline & 7 & 9 & 12 & 3 & 6 & 9 & 12 & 3 & 6 & 9 & 12 & 3 & \\
\hline 1. Project Planning & ॠ & & & & & & & & & & & & \\
\hline 2. Equipment & & खis & 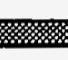 & 络 & & & & & & & & & \\
\hline 3. Hydrodynamic Tests & & & & ᄃ & & & & & & & & & \\
\hline 4. Beneficiation Tests & & & & & & & [ & & & & & & \\
\hline 5. Conventional Column Tests & & & & & & & & & E & & & & \\
\hline 6. Data Analysis & & & & $\sqsubset$ & & & & & & & & & \\
\hline 7. Reports & & $\square$ & $\square$ & $\square$ & $\square$ & $\square$ & 䀡 & $\square$ & $\square$ & $\square$ & 圆 & $\square$ & ; \\
\hline
\end{tabular}

Notes: $\quad \boldsymbol{\square}=$ Quarterly Technical Progress Report;
= Annual Report;
$\boldsymbol{\theta}=$ Final Report. 


\subsection{TECHINICAL PROGRESS}

\subsection{Task 2: Equipment Design and Construction}

The equipment design and constrution have been completed. Shake-down tests in the new column have also been carried out. Both liquid and gas flowmeters, as well as agitation speed controller have been calibrated. U-tube manometer system has been tested.

\subsection{Task 3: Hydrodynamic Tests}

\subsubsection{Objective}

The objective of this task is to pursue a basic understanding of the hydrodynamic behavior and to characterize the flow and mixing conditions in the new column. In this quarter, we have started to measure the overall gas holdup in the three-stage column.

\subsubsection{Principles}

Gas hold-up is one of the key parameters for evaluating the operating performance of the multistage flotation column. The overall gas hold-up $(\phi)$ is defined as:

$$
\Phi=\frac{V_{G}}{V_{G}+V_{L}}=\frac{V_{G L}-V_{L}}{V_{G L}}=\frac{H_{G L}-H_{L}}{H_{G L}}
$$

where $V_{G}$ is the volume of gas; $V_{G L}$ is the volume of liquid-gas layer; $V_{L}$ is the volume of liquid without gas bubble; $\mathrm{H}_{\mathrm{GL}}$ is the height of liquid-gas layer under bubbling conditions; and $\mathrm{H}_{\mathrm{L}}$ is the height of liquid level without gas bubbles. Thus, the overall gas holdup can be directly determined by volume expansion of liquid phase. 
The overall gas holdup can also be determined by measuring hydrostatic pressire difference at two positions along the column. A U-tube manometer (shown in Figure 2.1) was used for this purpose. If gas density is neglected, the relation between gas holdup and manometer reading can be expressed as:

$$
\Phi=\frac{H_{b}-\rho_{m} H_{m}-H_{w}}{H_{b}}
$$

where $\mathrm{H}_{b}$ denotes the liquid height in the column under bubbling condition; $\mathrm{H}_{\mathrm{w}}$ is the liquid height in the connecting tube; $\mathrm{H}_{\mathrm{m}}$ is the manometer reading; $\rho_{\mathrm{m}}$ is the density of the manometer liquid (see Figure 2.1).

\subsubsection{Experimental Setup and Procedure}

The experiments were performed in the three-stage agitated column in a semi-continuous mode. The flow diagram of this column has been presented in the last Quarterly Technical Progress Report ${ }^{[1]}$. The column consists of a plexiglass column of $0.1-\mathrm{m}$ diameter and $1.83-\mathrm{m}$ height. In the column, three vortex-inducing contactors are installed to create multiple loop flows. Within each contactor there are two impellers. The diameter ratio of contactor to column is 0.75 . A U-tube manometer is provided for measuring overall gas holdup.

Tap water was used as the liquid phase and air as the gas phase. In each run, a predetermined amount of tap water was added to the column and its initial volume (or height) was recorded. Air was then introduced into the bottom of the column through three porous ceramic spargers (mean pore size of $25 \mu \mathrm{m}$ ). The gas flow rate was controlled by a flowmeter. The 
superficial gas velocity (SGV) ranged from 0.5 to $8 \mathrm{~cm} / \mathrm{s}$. The agitation speed was varied over a range up to $45 \mathrm{~Hz}$. The frother used in this study was 4-methyl-2-pentanol (or methyl isobutyl carbinol, MIBC). MIBC was added to the tap water at various dosages up to $25 \mathrm{ppm}$. The frother was premixed with water in the column prior to start-up. All tests were carried out under ambient temperature and pressure. Table 2-1 lists the range of the key operating conditions employed in this study.

Table 2-1 Operating Conditions

\begin{tabular}{|l|l|}
\hline \multicolumn{1}{|c|}{ Parameters } & Conditions \\
\hline Superficial gas velocity, $10^{-2} \mathrm{~m} / \mathrm{s}$ & $0.5-8.0$ \\
\hline Agitation Speed, $\mathrm{Hz}$ & $0.0-45.0$ \\
\hline MIBC dosage, $\mathrm{mg} / \mathrm{L}$ & $0.0-25.0$ \\
\hline
\end{tabular}

\subsubsection{Results and Discussion}

A series of experiments on overall gas holdup of the multistage agitated column were conducted using both hydrostatic pressure difference method and volume expansion method. Our comparative test results confirmed that the gas holdup data determined by the direct measurement of liquid levels (see Eq. 2.2.1) is in good agreement with those obtained from measuring the hydrostatic pressures (see Eq. 2.2.2). The relative deviation of the data obtained from the two methods are less than 10\% (see Table 2-2). Chisti has reported similar results for evaluating these two methods ${ }^{[2]}$. All the data presented in this report are obtained using the hydrostatic pressure difference method (see Figure 2-1). 
Table 2-2 Comparison of the Gas Holdup Data Obtained from Two Different Methods

\begin{tabular}{|c|ccccccccc|}
\hline Method & \multicolumn{7}{|c|}{ Overall gas holdup } \\
\hline Volume expansion & 0.08 & 0.085 & 0.093 & 0.110 & 0.130 & 0.285 & 0.296 & 0.318 & 0.364 \\
\hline Pressure difference & 0.08 & 0.085 & 0.092 & 0.109 & 0.126 & 0.261 & 0.271 & 0.293 & 0.340 \\
\hline
\end{tabular}

Note: SGV : $1.76-7.63 \mathrm{~cm} / \mathrm{s}$; Agitation speed : 0 - $45 \mathrm{~Hz} ; \mathrm{MBC}$ dosage: $7 \mathrm{mg} / \mathrm{L}$

The effects of SGV, agitation speed and MIBC dosage on overall gas holdup have been investigated. The experimental data for the roles of these operating parameters were plotted in a three dimension axes' system as shown in Figures 2-2 to 2-6.

First, Figures 2-2 to 2-6 show the effect of SGV on the gas holdup. The data indicate that when the SGV is less than $0.06 \mathrm{~m} / \mathrm{s}$, the gas holdup increases significantly with an increase in SGV. Once the SGV becomes greater than $0.06 \mathrm{~m} / \mathrm{s}$, the increase in gas holdup with SGV apparently slows down. Based on our observation, this corresponds to a visual change in the flow regime. In the first regime, gas stream with uniform large- size bubbles entered into the contacting region and the bubbles break up into small bubbles under agitation. As the SGV increased, loop-flow around the contactors was intensified. In this case, the bubbles became smaller and led to increasing the gas holdup. When the SGV increased beyond $0.06 \mathrm{~m} / \mathrm{s}$, there was a more rapid increase in the turbulence. Consequently, an emulsion flow pattern appeared around the three contactors. This corresponds to the second flow regime. In this regime, the gas holdup behaviour is quite different from the first flow regime. On one hand, the vortex turbulent motion caused by the loop flow in the multistage contactors did enhance the bubble break up and inhibit, to some extent, the effect of 
bubble coalescence; thus, relatively uniform fine bubbles filled the entire column volume which in turn leads to a high gas holdup. On the other hand, after gas bubble size is reduced to a limiting size range (i.e. the bubble breakup rate is equal to the bubble coalescence rate) the rate of increase in gas holdup declines with increasing SGV.

The agitation speed is another important operating parameter affecting the gas holdup. As shown in Figure 2-2 through 2-6, a similar inflection in the increase of the gas holdup with increasing agitation speed is noted at various SGV levels. This phenomenon also corresponds to a visually observed change in the flow regime as dicussed in the previous paragraph. The initial gradual increase in gas holdup with increasing agitation speed quickly gives way to a more rapid rise of gas holdup when the agitation speed is beyond $14.5 \mathrm{~Hz}$.

Since frother could be expected to alter bubble coalescence and breakup, the effect of such agents on gas holdup was also studied. The effect of MIBC dosage on the gas holdup under varying SGV and agitation speed can be seen in analysing the three dimension plots from Figures 2-2 through 2-6. The results indicate that the MBBC concentration did have a significant effect on the gas holdup at higher SGVs. These phenomena may be attributed to the fact that at higher SGVs, a greater surface activity would result in the presence of a frother. This in turn would reduce coalescence and lead to higher gas holdup. It is also noted that as the MIBC dosage increases beyond $15 \mathrm{mg} / \mathrm{L}$, the rate of increase in gas holdup tends to decline ( see Figures 2-4 through 2-6). This is also consistent with the visually observed change in flow regime. 


\subsubsection{Summary}

Experimental measurements of the overall gas holdup were conducted to examine the roles of various operating parameters in the multistage agitated column operation. The results show that

1. The relationship of the gas holdup with the SGV or agitation speed can be used to define the two flow regimes in a multistage agitated column. The experimental results revealed that the inflection point on the curves (i.e., near the SGV of about $0.06 \mathrm{~ms}^{-1}$ and agitation speed beyond $14.5 \mathrm{~Hz}$ ) corresponds to a visually observed change in the flow regime from bubble flow to emulsion flow.

2. In first flow regime, the SGV plays more important role than agitation speed in increasing gas holdup. However, in the second flow regime, the effect of agitation speed on gas holdup is more significant than that of SGV.

3. The effect of frother dosage on gas holdup was marked in the second flow regime. However, when frother dosage is greater than $15 \mathrm{mg} / \mathrm{L}$, the increase in gas holdup levels off.

\subsection{WORK FORECAST}

In the next quarter, the following tasks will be performed:

1. Continue to carry out gas holdup measurement:

a. Overall gas holdup in a conventional flotation column (without vortex-inducing contactor);

b. Local gas holdup in the multistage agitated column and conventional flotation column. 
2. Initiate investigation of mixing and liquid circulation behaviors :

a. Set up and calibrate a conductivity device;

b. Measure the mixing time;

c. Investigate the liquid circulation behaviour around the contactors.

The geometric dimension and the range of operating parameters to be used in the conventional flotation column will be the same as those employed in the multistage agitated column. Thus, a direct comparison between the results obtained from the two columns can be made.

\subsection{REFERENCES}

1. Chiang, S.H., "A Study of Multistage/Multifunction Column for Fine Particle Separation", Quarterly Technical Progress Report, (July 8, 1994 - September 30, 1994), Submitted to U.S. Department of Energy, PETC, January 20, 1995.

2. Chisti, M. Y., <Airlift Bioreactors>, Elsevier Applied Science, London and New York, 1989. 


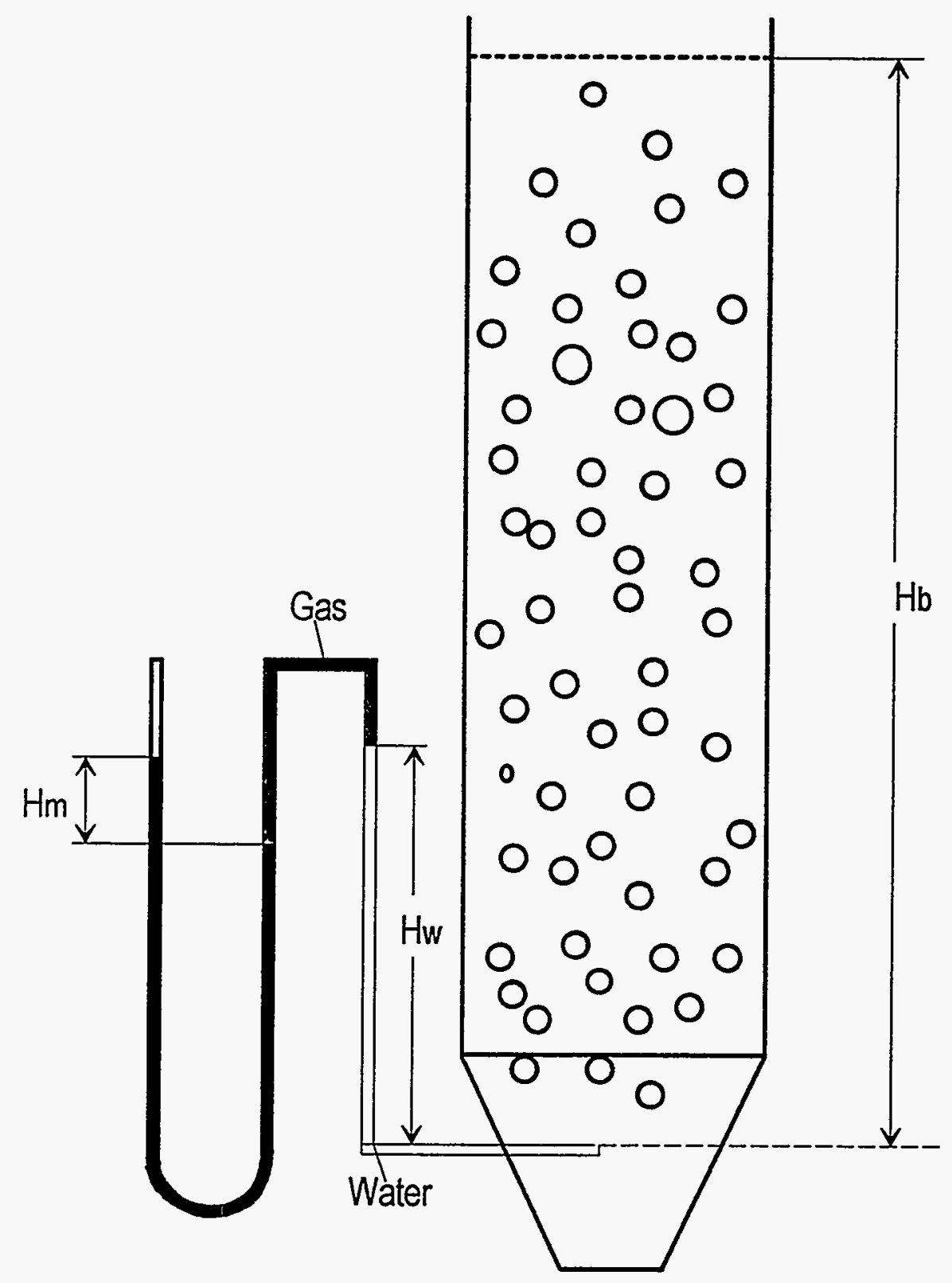

Figure 2-1 U-tube manometer setup for gas holdup measurement 


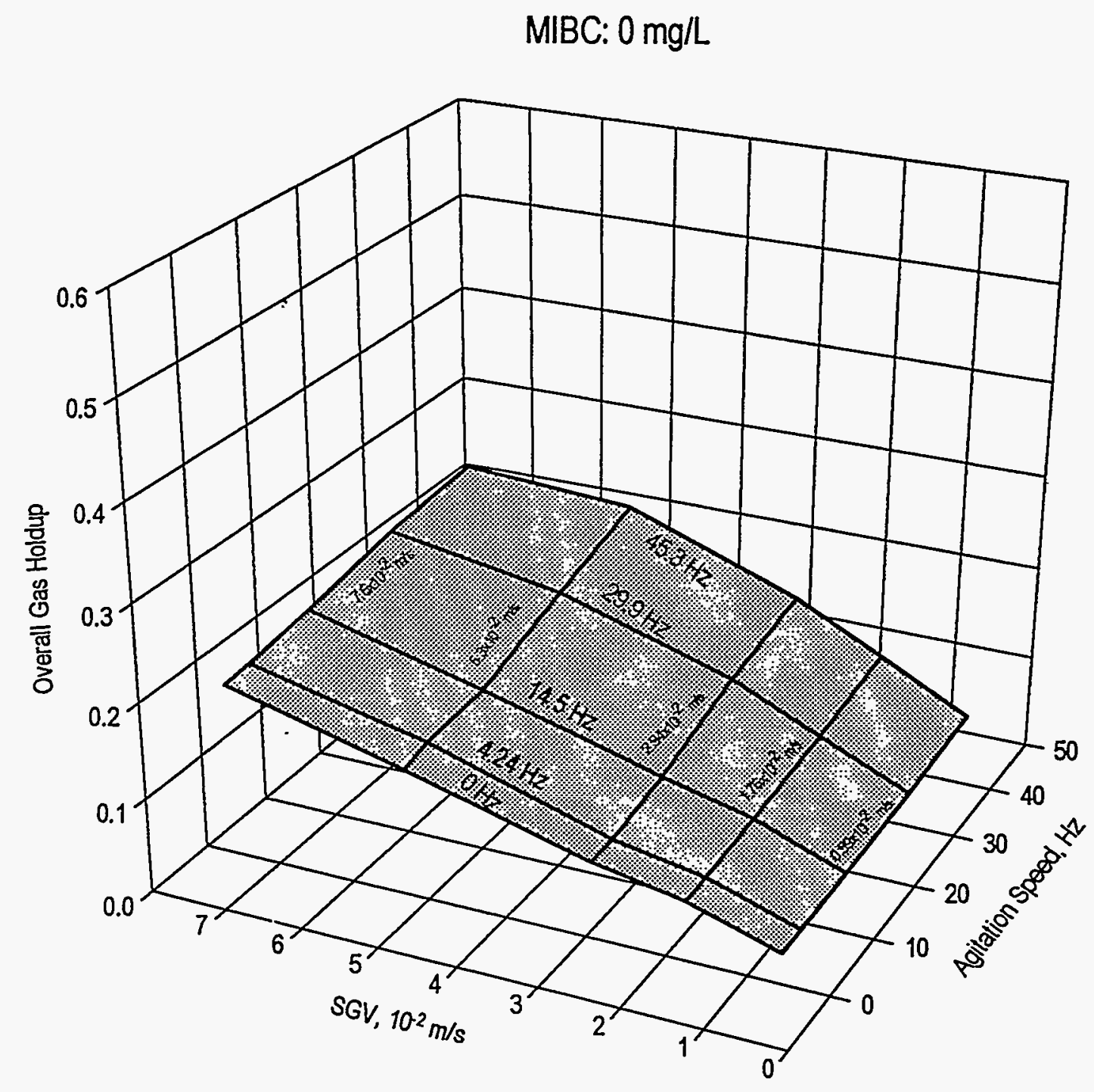

Figure 2-2 Effects of SGV and Agitation Speed on Gas Holdup 


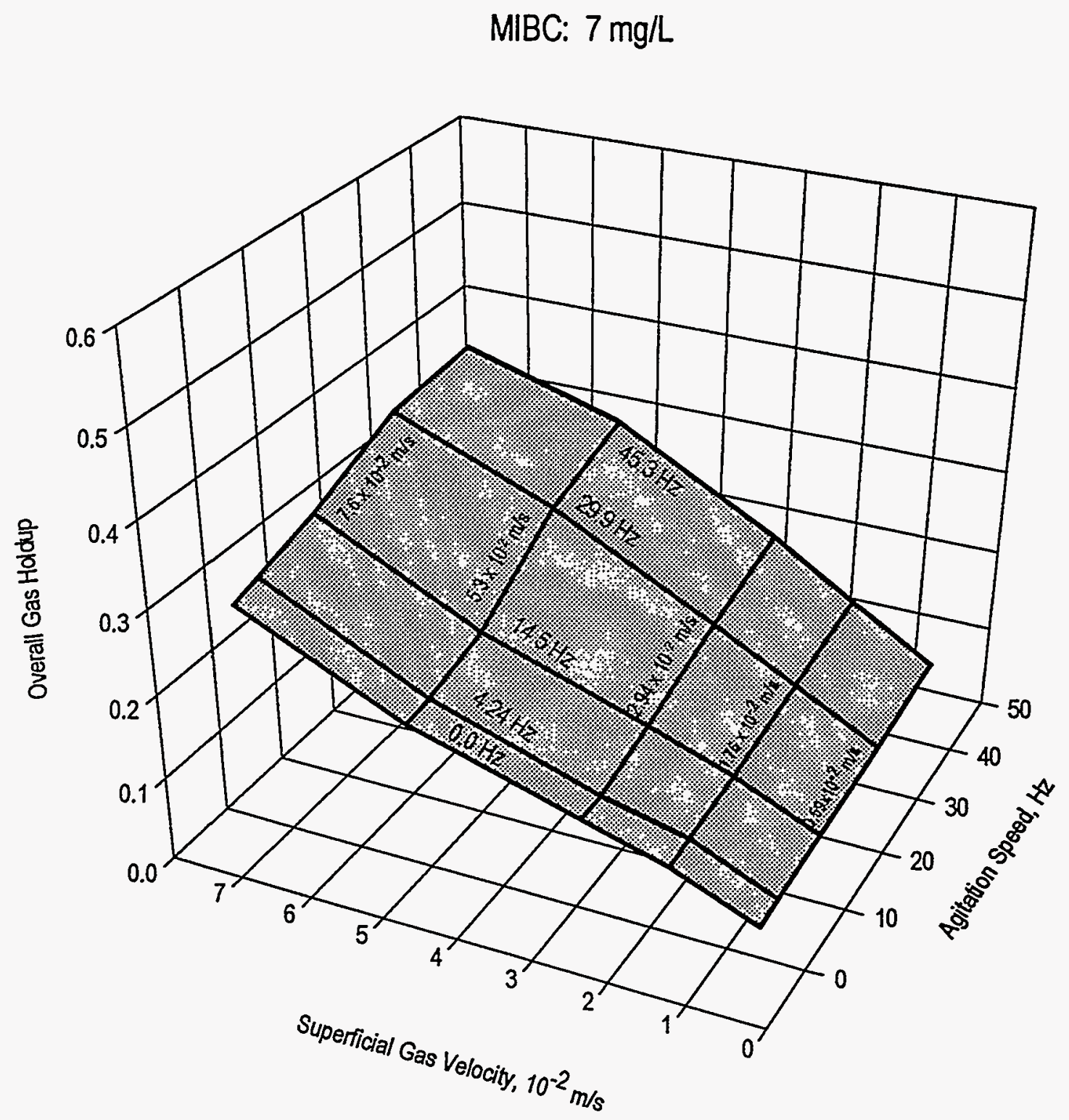

Figure 2-3 Effects of SGV and Agitation Speed on Gas Holdup 


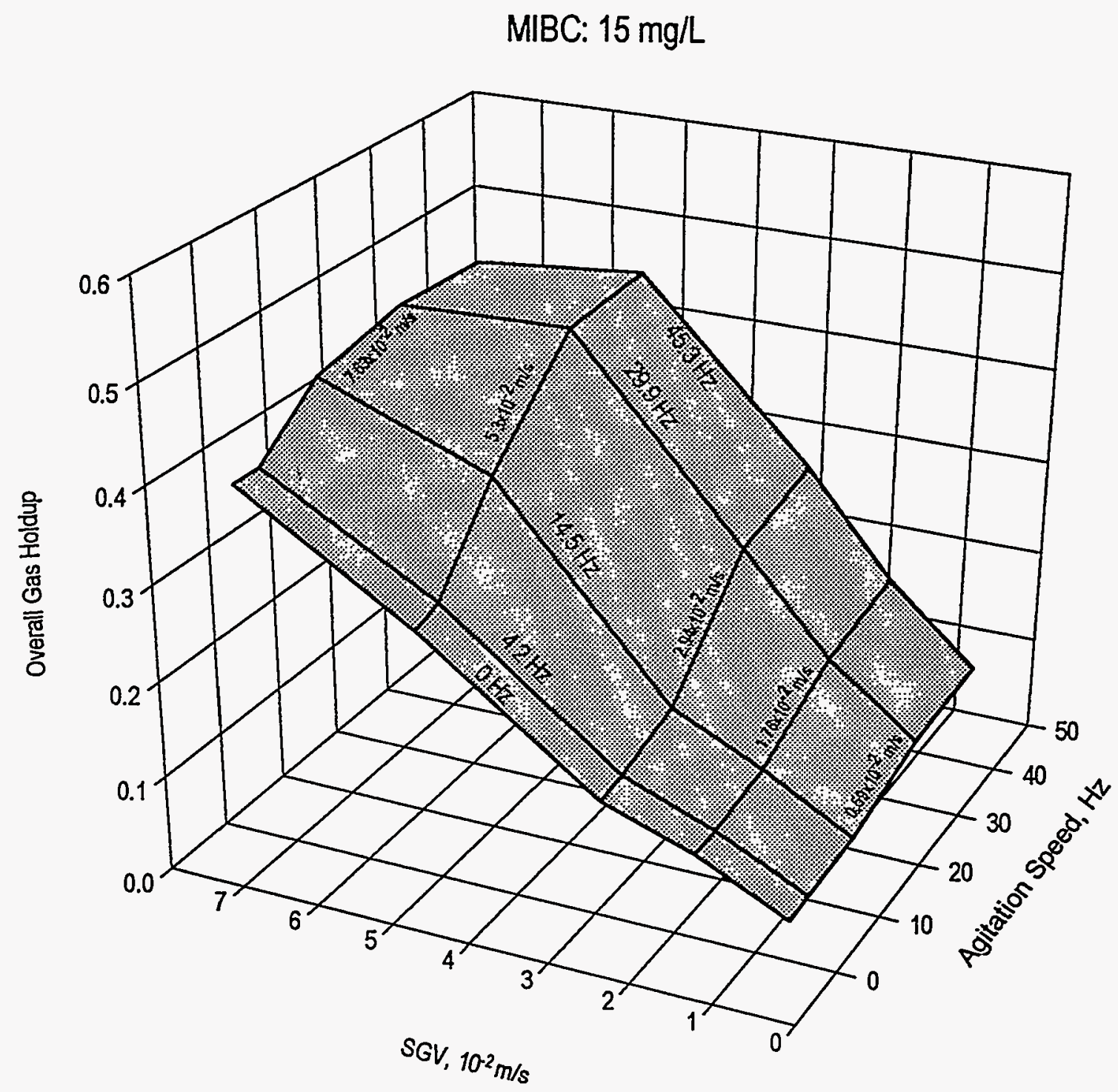

Figure 2-4 Effect of SGV and Agitation Speed on Gas Holdup 


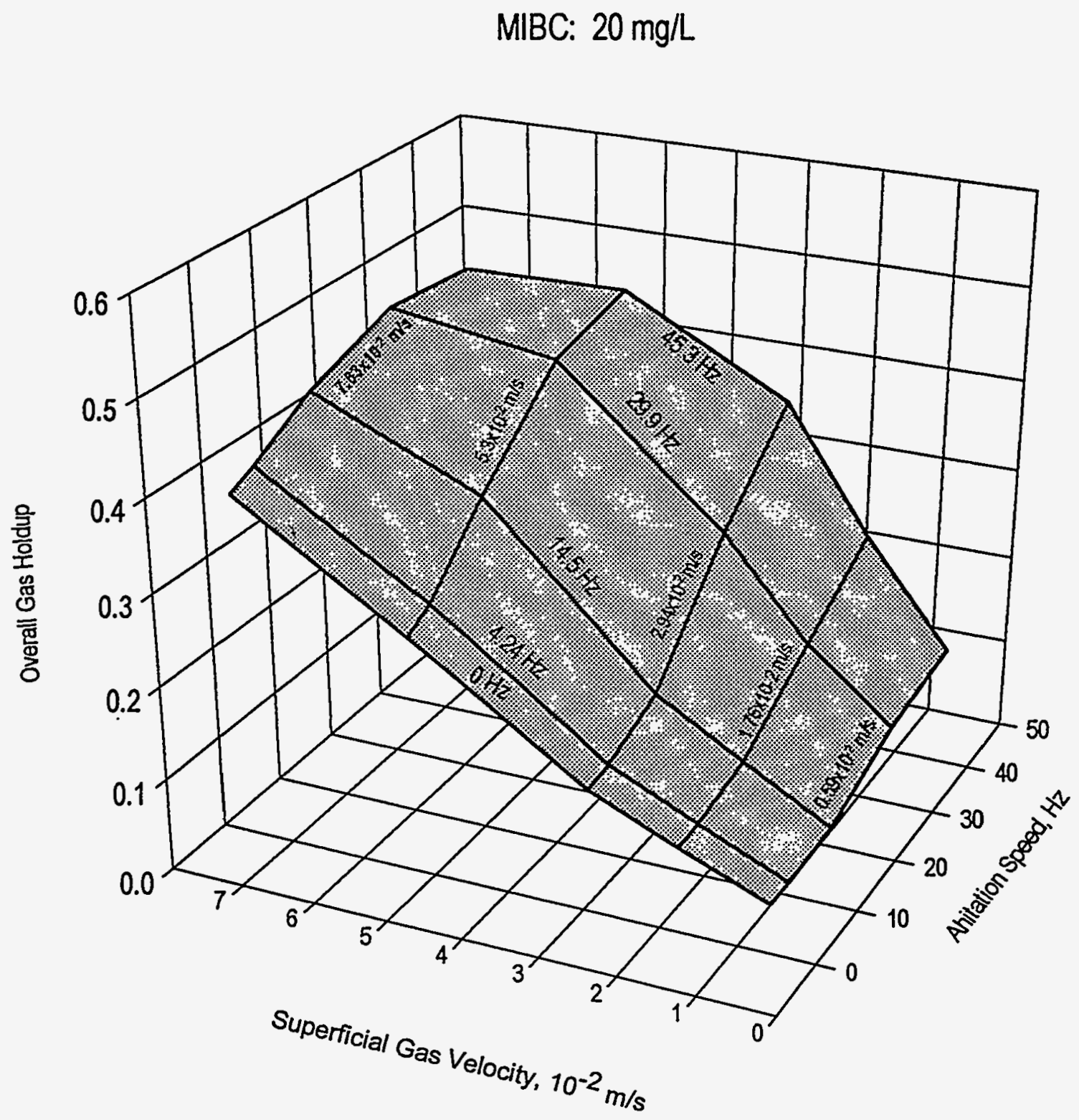

Figure 2-5 Effects of SGV and Aigitation Speed on Gas Holdup 


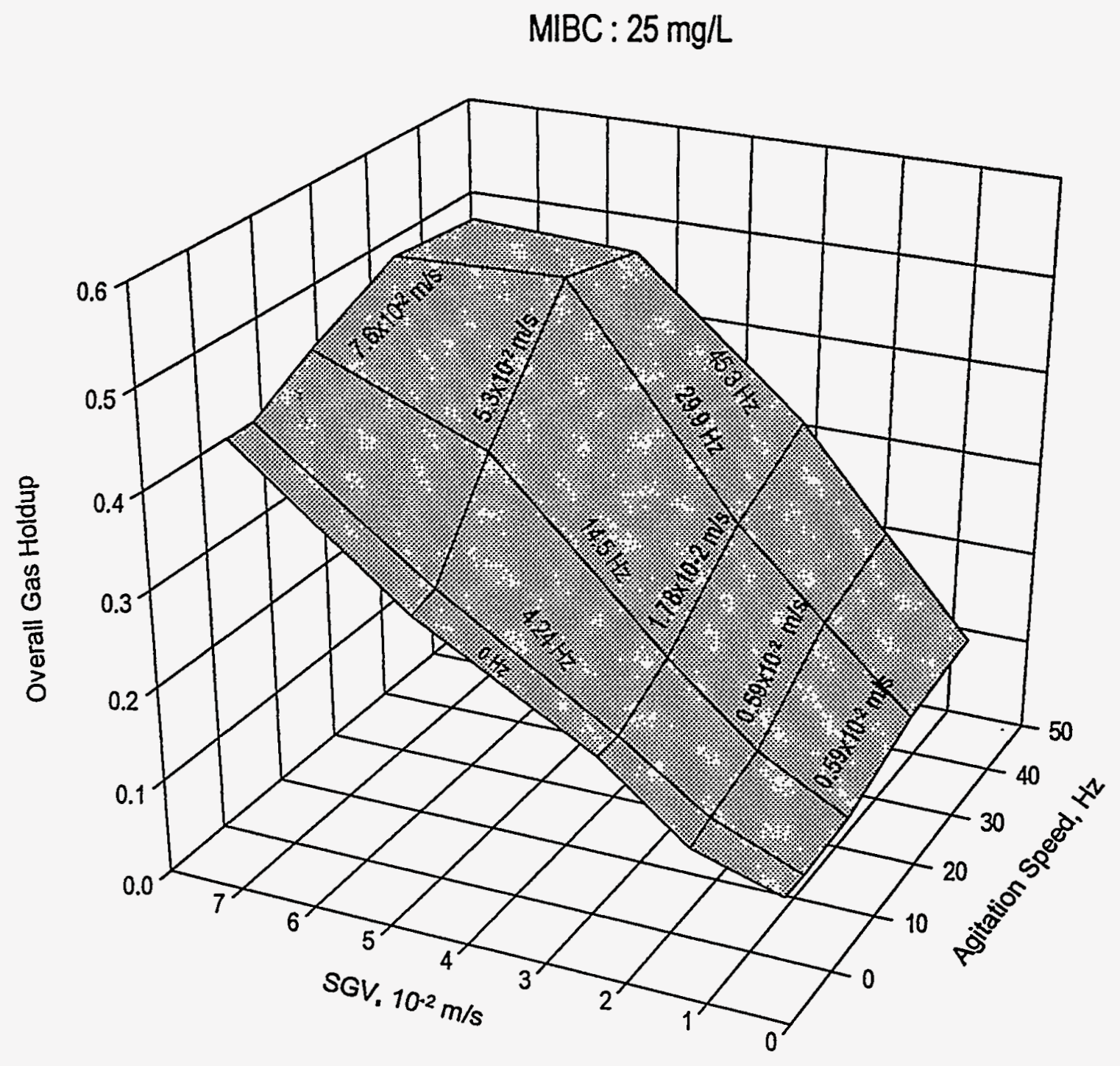

Figure 2-6 Effects of SGV and Agitation Speed on Gas Holdup 